\title{
The association between socioeconomic disparities and left ventricular hypertrophy in chronic kidney disease: results from the KoreaN Cohort Study for Outcomes in Patients With Chronic Kidney Disease (KNOW-CKD)
}

Eunjeong Kang ${ }^{1}$, Joongyub Lee ${ }^{2,3}$, Hyo Jin Kim ${ }^{4}$, Miyeun Han ${ }^{5}$, Soo Wan Kim6, Kyu-Beck Lee ${ }^{7}$, Suah Sung ${ }^{8}$, Tae-Hyun Yoo', Wookyung Chung ${ }^{10}$, Curie Ahn ${ }^{1}$ and Kook-Hwan Oh' ${ }^{1 *}$ (D)

\begin{abstract}
Background: Left ventricular hypertrophy (LVH) is one of the risk factors for cardiovascular (CV) disease and mortality. However, the relationship between socioeconomic status (SES) and LVH in chronic kidney disease remains unclear.

Methods: Data were collected from the KoreaN Cohort Study for Outcome in Patients With Chronic Kidney Disease (KNOW-CKD, NCT01630486 at http://www.clinicaltrials.gov). Subjects with CKD and aged $\geq 50$ were included. SES was characterized based on monthly income and educational attainment, each of which was divided into three strata. LVH was defined as LV mass/height ${ }^{2.7} \geq 47 \mathrm{~g} / \mathrm{m}^{2.7}$ in female and $\geq 50 \mathrm{~g} / \mathrm{m}^{2.7}$ in male. Age, sex, diabetes, CKD stage, body mass index, blood pressure and physical activity were included as covariates.

Results: A total of 1361 patients were included. Mean age was $60.9 \pm 6.9$ years, and 63.2\% were men. Higher education level was associated with higher monthly income ( $P$ for trend $<0.001)$. The lowest education level was independently associated with LVH (lower than high school, adjusted odds ratio [OR] 1.485, 95\% Cl 1.069-2.063, $P=0.018$; completed high school, adjusted OR 1.150, 95\% confidence interval [CI] 0.834-1.584, $P=0.394$; highest education level as the reference). Monthly income level was marginally associated with $\mathrm{LVH}$ after adjusting for covariates (\$1500-4500, adjusted OR 1.230, 95\% Cl 0.866-1.748, $P=0.247$; $<\$ 1500$, adjusted OR 1.471, 95\% Cl 1.002-2.158, $P=0.049 ;>\$ 4500$; reference).

Conclusions: In the CKD population, lower SES, defined by educational attainment and low income level exhibited a significant association with LVH, respectively. Longitudinal follow-up will reveal whether lower SES is associated with poor CKD outcomes.
\end{abstract}

Keywords: Chronic kidney disease, Left ventricular hypertrophy, Education, Income, Socioeconomic status

\footnotetext{
*Correspondence: khoh@snu.ac.kr; ohchris@hanmail.net

The results presented in this paper have not been published previously in

whole or part, except in abstract form.

${ }^{1}$ Department of Internal Medicine, Seoul National University College of

Medicine, Seoul, Korea

Full list of author information is available at the end of the article
}

(0) The Author(s). 2018, corrected publication September 2018. Open Access This article is distributed under the terms of the Creative Commons Attribution 4.0 International License (http://creativecommons.org/licenses/by/4.0/), which permits

unrestricted use, distribution, and reproduction in any medium, provided you give appropriate credit to the original author(s) and the source, provide a link to the Creative Commons license, and indicate if changes were made. The Creative Commons Public Domain Dedication waiver (http://creativecommons.org/publicdomain/zero/1.0/) applies to the data made available in this article, unless otherwise stated. 


\section{Backgrounds}

Socioeconomic status (SES) is an important and strong predictor of morbidity and mortality. [1] Generally, education level, occupation, race, housing, social support and income are key components to be evaluated as SES. Multiple determinants of health care level vary with SES levels, including risk of all-cause mortality, [2] cardiovascular diseases, [3-5] diabetes mellitus, [5, 6] cancer, $[7,8]$ and chronic kidney disease (CKD) [5, 9]. The reasons behind this phenomenon have been suggested as follows: educational attainment and income levels contribute to a complex set of socio-economic determinants, including insurance, transportation, stress, housing quality and access to health care [10]. Such determinants may interact and combine to affect the health outcomes in an interconnected mechanism. Because SES appears to affect CKD patients in a similar way as it does the general population, it is important to clarify the health-related risk factors in CKD influenced by SES.

Worldwide, cardiovascular diseases are the leading cause of death among patients with CKD and end-stage renal disease (ESRD). Cardiovascular mortality is the primary cause of death in CKD patients in Korea, accounting for the $39 \%$ of mortality cases in peritoneal dialysis and $36 \%$ in hemodialysis patients [11]. Thus, most physicians make every effort to prevent a cardiovascular event and control its risk factors, such as lipid levels and anemia. Above all, left ventricular hypertrophy (LVH) causes decreased diastolic compliance and leads to ischemic cardiomyopathy, even in the absence of coronary artery disease [12]. More specifically, in a cohort of patients starting dialysis therapy, cardiac enlargement and decreased systolic function exhibited a relationship with ischemic heart disease and cardiac failure [13].

Meanwhile, in a previous study, Carlos et al. [14] reported that lower SES is an independent risk factor for increased left ventricular mass among hypertensive and normotensive African Americans. However, the relationship between SES and risk factors of cardiovascular mortality, including left ventricular hypertrophy ( $\mathrm{LVH})$, are less well known among CKD patients.

Since some social determinants of health are modifiable through education and governmental health policies, investigating the influence of SES on the outcome of CKD is crucial. Therefore, we investigated the association between $\mathrm{LVH}$, the representative risk factor for $\mathrm{CV}$ mortality in CKD and socioeconomic status, evaluated by educational attainment and monthly income level, among participants in the KoreaN cohort study for Outcome in patients With Chronic Kidney Disease (KNOW-CKD).

\section{Methods}

\section{Study population}

Participants in the KNOW-CKD, a Korean multicenter prospective cohort study that enrolled subjects with CKD from stage 1 to 5 (predialysis) from June 30, 2011 to January 29, 2016, were included in this cross-sectional analysis. The detailed design and methods of the KNOW-CKD have been previously published elsewhere [15]. In total, 2238 participants were enrolled in the KNOW-CKD study. Among them, we excluded individuals who did not respond to the questionnaire regarding SES, no measured left ventricular (LV) mass, and who were aged $<50$ years (Fig. 1). Finally, 1330 subjects were included in the analyses.

\section{Variable measurements}

Demographics, and clinical and laboratory values at enrollment were extracted from an electronic data management system (http://www.phactaX.org). The estimated glomerular filtration rate (eGFR) was estimated using the Chronic Kidney Disease Epidemiology Collaboration (CKD-EPI) equation using creatinine [16]. Resting blood pressure was measured with mercury sphygmomanometers and cuffs of appropriate size three times for average blood pressure. Hypertension (HTN) was defined as a blood pressure recording $\geq 140 / 90 \mathrm{mmHg}$, a self-reported history of hypertension, or use of antihypertensive agents. Diabetes (DM) was defined by self-reporting or use of hypoglycemic medications. Physical activity was quantified by the International Physical Activity Questionnaire. Subjects were categorized by total Metabolic Equivalent of Task (MET) - minutes/ week; "high" was defined as $\geq 3000$ METs-minutes/week, "moderate" as 600-2999 METs-minutes/week and "low" as $<600$ METs-minutes/week. Anemia was defined as hemoglobin $<13 \mathrm{~g} / \mathrm{dL}$ for males, or $<12 \mathrm{~g} / \mathrm{dL}$ for females. Two-dimensional echocardiography was conducted to measure cardiac parameters. LV mass was calculated by 0.8 $\mathrm{x}\left\{1.04\left[(\text { LVIDd + PWTd + SWTd })^{3}-(\text { LVIDd })^{3}\right]\right\}+0.6$ g, where PWTd and SWTd are posterior wall thickness at end diastole and septal wall thickness at end diastole, respectively [17]. Left ventricular hypertrophy (LVH) was defined as LV mass $/$ height $^{2.7} \geq 47 \mathrm{~g} / \mathrm{m}^{2.7}$ in female and $\geq 50 \mathrm{~g} /$ $\mathrm{m}^{2.7}$ in male, $[17,18]$ because LV mass indexed to body surface area is problematic in that weight is affected by volume overload in CKD [19].

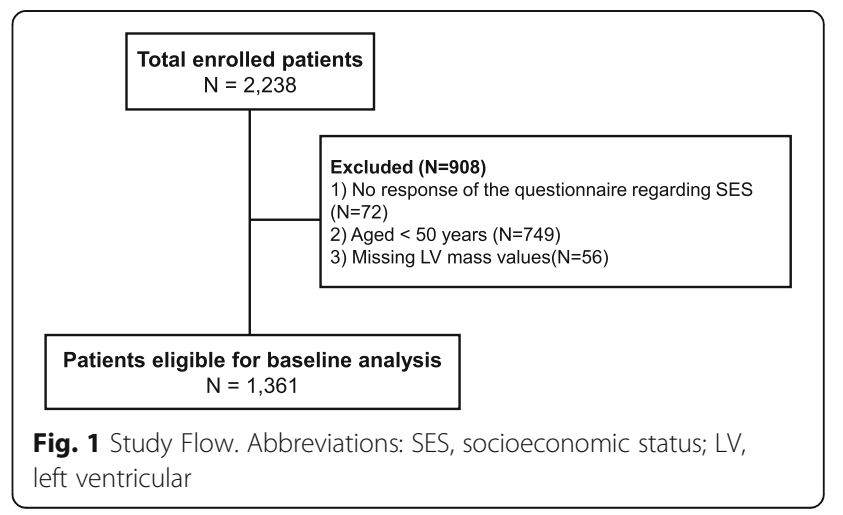




\section{Evaluation of socioeconomic status}

The questionnaire used in the KNOW-CKD Study followed those of the Korea National Health and Nutrition Examination Survey (KNHANES). The KNAHES classified the monthly income of Korean population into three intervals, based on the average household income of the contemporary Koreans. Information on educational attainment and monthly income level, which were captured from patient-reported questionnaire, was used as indicators of SES. With regard to educational attainment, the patients were asked about the level at which their formal school education was completed. Educational attainment was classified into three levels: "less than high school" included those who never went to high school or who completed only part of high school, "completed high school" included those who had graduated high school but not completed college, "college degree or beyond" included those who had completed college or a higher degree. Monthly income level was classified into three levels: less than \$ 1500, \$ 1500 to \$ 4500 , and over $\$ 4500$ per month.

\section{Statistical analysis}

Continuous variables are presented as a mean \pm standard deviation. Proportions were used for categorical variables, including age groups, sex, CKD stages, comorbidities (e.g., diabetes mellitus). We used one-way analysis of variance for comparison of continuous variables and the $X^{2}$ test for categorical variables. Statistical significance was determined at $P<0.05$ using two-sided tests. We conducted logistic regression to evaluate the association between LVH and SES, which is categorized into three education or monthly income levels. We checked the Hosmer-Lemeshow goodness of fit test for determining whether multivariable models are fit of data. Statistical analyses were carried out using the SPSS software package, version 22.0 (IBM Corporation, Armonk, NY, USA).

\section{Ethics statement}

The study protocol was approved by the Institutional Review Board at each participating clinical center - i.e., Seoul National University Hospital (1104-089-359), Seoul National University Bundang Hospital (B-1106/129-008), Yonsei University Severance Hospital (4-2011-0163), Kangbuk Samsung Medical Center (2011-01-076), Seoul St. Mary's Hospital (KC11OIMI0441), Gil Hospital (GIRBA2553), Eulji General Hospital (201105-01), Chonnam National University Hospital (CNUH-2011-092), and Pusan Paik Hospital (11-091) in 2011. This study was conducted in accordance with the principles of the Declaration of Helsinki.

\section{Results}

Baseline characteristics of study participants

The baseline characteristics of the study subjects are shown in Table 1. Mean age was $60.9 \pm 6.9$ years, and 860
(63.2\%) were men. Mean eGFR was $45.5 \pm 25.1 \mathrm{~mL} / \mathrm{min} /$ $1.73 \mathrm{~m}^{2}$ and the number of patients with a CKD stage G3a, G3b, G4, and G5 were 226 (16.6\%), 250 (18.4\%), 322 (23.7\%), 352 (25.9\%) and 1023 (75.1\%), respectively. Diabetic nephropathy was the most common cause of CKD (31.8\%), followed by glomerulonephritis (25.3\%), hypertensive nephropathy (24.2\%), and polycystic kidney disease (10.4\%). Subjects with DM and HTN comprised 42.0\% and $98.0 \%$ of the study participants, respectively. Anemia was prevalent in 662 (49.1\%) subjects and 119 (8.7\%) subjects received erythropoiesis-stimulating agents (ESAs).

\section{Comparison of baseline characteristics according to socioeconomic status}

We compared baseline characteristics according to educational attainment (Table 1) and monthly income levels (Table 2). With respect to educational attainment strata, the mean age was 2 years older in the lowest educational group. Subjects in the lowest education group exhibited the lowest eGFR ( $47.1 \pm 23.3$ for 'college or beyond', 46.7 \pm 26.6 for 'completed high school', and $42.7 \pm 25.0 \mathrm{~mL} /$ $\mathrm{min} / 1.73 \mathrm{~m}^{2}$ for 'less than high school' groups, respectively; $P$ for trend $=0.008$ ). The higher education group was associated with higher monthly family income ( $P$ for trend $<0.001)$. The prevalence of anemia increased with decreasing level of educational attainment $(41.2 \%, 48.8 \%$, and $57.0 \%$, respectively; $P$ for trend $<0.001$ ). The lowest educational attainment was an independent risk factor for anemia, even after adjusting for age, sex, and eGFR ('less than high school', OR 1.515, 95\% CI 1.075-2.136, $P=0.018)$. The proportion of diabetic patients was the highest in the lowest education group. No group differences were exhibited in sodium excretion from 24-h urine collection.

When the subjects were categorized based on monthly income level, similar trends were observed in terms of age, eGFR, DM, anemia and 24-h sodium excretion (Table 2).

\section{Socioeconomic status and left ventricular hypertrophy}

The total number of patients diagnosed with LVH on echocardiography was 413 (30.3\%). With the increase of the household income level or with the increase of the educational level, the prevalence of LVH gradually increased (Tables 1 and 2, $P$ for trend $<0.001$, respectively). In unadjusted analyses, risk of LVH increased with decreasing levels of educational attainment and monthly income level, respectively (Table 3, Table 4, and Fig. 2). In particular, the lowest educational level (lower than high school) was independently associated with LVH after adjusting for age, sex, body mass index, mean arterial pressure, DM, CKD stage and physical activity ('college or beyond', reference; 'completed high school', OR 1.150, 95\% CI 0.834-1.584; 'less than high school' OR 1.485, 95\% CI 1.069-2.063; Fig. 3). Additionally, 
Table 1 Baseline Characteristics according to Educational attainment

\begin{tabular}{|c|c|c|c|c|c|c|}
\hline \multirow[t]{3}{*}{ N } & \multirow{3}{*}{$\begin{array}{c}\text { Total } \\
1361 \\
\end{array}$} & \multicolumn{3}{|c|}{ Educational attainment } & \multirow[t]{3}{*}{$P$} & \multirow{3}{*}{$\begin{array}{l}P \text { for } \\
\text { trend }\end{array}$} \\
\hline & & $\begin{array}{l}\text { College or } \\
\text { beyond }\end{array}$ & $\begin{array}{l}\text { Completed } \\
\text { High school }\end{array}$ & $\begin{array}{l}\text { Lower than } \\
\text { high school }\end{array}$ & & \\
\hline & & 437 & 467 & 457 & & \\
\hline Age (year, mean $\pm S D$ ) & $60.9 \pm 6.9$ & $60.3 \pm 7.1$ & $60.0 \pm 6.9$ & $62.4 \pm 6.6$ & $<0.001$ & $<0.001$ \\
\hline Male (N, \%) & $860(63.2)$ & $361(82.6)$ & $299(64.0)$ & $200(43.8)$ & $<0.001$ & $<0.001$ \\
\hline LVH (N, \%) & $413(30.3)$ & $101(23.1)$ & $132(28.3)$ & $180(39.4)$ & $<0.001$ & $<0.001$ \\
\hline $\operatorname{eGFR}\left(\mathrm{mL} / \mathrm{min} / 1.73 \mathrm{~m}^{2}\right)$ & $45.5 \pm 25.1$ & $47.1 \pm 23.3$ & $46.7 \pm 26.6$ & $42.7 \pm 25.0$ & $0.001^{\mathrm{a}}$ & 0.008 \\
\hline $\mathrm{BMI}\left(\mathrm{kg} / \mathrm{m}^{2}\right.$, mean $\left.\pm \mathrm{SD}\right)$ & $24.6 \pm 3.1$ & $24.6 \pm 2.9$ & $24.4 \pm 3.2$ & $24.9 \pm 3.2$ & 0.049 & 0.155 \\
\hline \multicolumn{2}{|l|}{ CKD stage $(\mathrm{N}, \%)$} & & & & $<0.001$ & $<0.001$ \\
\hline Stage G1 & $112(8.2)$ & $27(6.2)$ & $50(10.7)$ & $35(7.7)$ & & \\
\hline Stage G2 & $226(16.6)$ & $89(20.3)$ & $72(15.4)$ & $65(14.2)$ & & \\
\hline Stage G3a & $250(18.4)$ & $107(24.5)$ & $83(17.8)$ & $60(13.1)$ & & \\
\hline Stage G3b & $322(23.7)$ & $90(20.6)$ & $112(24.0)$ & $120(26.3)$ & & \\
\hline Stage G4 & $352(25.9)$ & $101(23.1)$ & $114(24.4)$ & $137(30.0)$ & & \\
\hline Stage G5 & $99(7.3)$ & $23(5.3)$ & $36(7.7)$ & $40(8.8)$ & & \\
\hline \multicolumn{2}{|l|}{ Underlying renal disease $(\mathrm{N}, \%)$} & & & & 0.042 & 0.049 \\
\hline Diabetic nephropathy & $429(31.8)$ & $130(29.9)$ & $142(30.7)$ & $157(34.7)$ & & \\
\hline Hypertensive nephropathy & $327(24.2)$ & $109(25.1)$ & $105(22.7)$ & $113(25.0)$ & & \\
\hline Glomerulonephritis & $341(25.3)$ & $104(23.9)$ & $136(29.4)$ & $101(22.3)$ & & \\
\hline Polycystic kidney disease & $140(10.4)$ & $60(13.8)$ & $42(9.1)$ & $38(8.4)$ & & \\
\hline Diabetes mellitus (N, \%) & $571(42.0)$ & $168(38.4)$ & $189(40.5)$ & $214(46.8)$ & 0.022 & 0.019 \\
\hline bHemoglobin A1c (\%, mean \pm SD) & $6.9 \pm 1.4$ & $6.7 \pm 1.2$ & $6.9 \pm 1.3$ & $7.1 \pm 1.5$ & $0.005^{\mathrm{a}}$ & 0.001 \\
\hline Hypertension (N, \%) & $1334(98.0)$ & $430(98.4)$ & $457(97.9)$ & $447(97.8)$ & 0.673 & 0.635 \\
\hline Systolic blood pressure (mmHg, mean $\pm \mathrm{SD}$ ) & $128.84 \pm 16.7$ & $127.8 \pm 15.2$ & $129.1 \pm 17.3$ & $129.5 \pm 17.5$ & $0.539^{\mathrm{a}}$ & 0.138 \\
\hline Diastolic blood pressure $(\mathrm{mmHg}$, mean $\pm \mathrm{SD})$ & $75.9 \pm 11.2$ & $76.3 \pm 10.7$ & $76.6 \pm 10.9$ & $74.9 \pm 11.8$ & 0.071 & 0.068 \\
\hline \multicolumn{2}{|l|}{ Monthly income $(\mathrm{N}, \%)$} & & & & $<0.001$ & $<0.001$ \\
\hline$>\$ 4500$ & $269(19.8)$ & $163(37.3)$ & $73(15.6)$ & $33(7.2)$ & & \\
\hline$\$ 1500$ to 4500 & $701(51.5)$ & $214(49.0)$ & $279(59.7)$ & $208(45.5)$ & & \\
\hline$<\$ 1500$ & $391(28.7)$ & $60(13.7)$ & $115(24.6)$ & $216(47.3)$ & & \\
\hline \multicolumn{2}{|l|}{ Physical activity (N, \%) } & & & & $<0.001$ & $<0.001$ \\
\hline High & $287(21.1)$ & $95(21.7)$ & $112(24.0)$ & $80(17.5)$ & & \\
\hline Moderate & $536(39.4)$ & $201(46.0)$ & $170(36.4)$ & $165(36.1)$ & & \\
\hline Low & $538(39.5)$ & $141(32.3)$ & 185 (39.6) & $212(46.4)$ & & \\
\hline Anemia (N, \%) & $662(49.1)$ & $178(41.2)$ & $225(48.8)$ & $259(57.0)$ & $<0.001$ & $<0.001$ \\
\hline ESA $(N, \%)$ & $119(8.7)$ & $30(6.9)$ & $33(7.1)$ & $56(12.3)$ & 0.005 & 0.006 \\
\hline \multicolumn{7}{|l|}{ Serum laboratory values } \\
\hline Hemoglobin ( $\mathrm{g} / \mathrm{dL}$, mean $\pm \mathrm{SD}$ ) & $12.6 \pm 2.0$ & $13.2 \pm 2.0$ & $12.6 \pm 1.9$ & $12.0 \pm 1.92$ & $<0.001$ & $<0.001$ \\
\hline Creatinine $(\mathrm{mg} / \mathrm{dL}$, mean $\pm \mathrm{SD})$ & $1.9 \pm 1.1$ & $1.9 \pm 1.1$ & $1.9 \pm 1.1$ & $1.9 \pm 1.1$ & 0.986 & 0.869 \\
\hline Albumin $(\mathrm{g} / \mathrm{dL}$, mean $\pm \mathrm{SD})$ & $4.2 \pm 0.4$ & $4.2 \pm 0.4$ & $4.2 \pm 0.4$ & $4.1 \pm 0.4$ & 0.014 & 0.003 \\
\hline Calcium (mg/dL, mean \pm SD) & $9.1 \pm 0.5$ & $9.1 \pm 0.5$ & $9.1 \pm 0.5$ & $9.1 \pm 0.5$ & 0.413 & 0.206 \\
\hline Phosphorus (mg/dL, mean \pm SD) & $3.7 \pm 0.7$ & $3.6 \pm 0.6$ & $3.7 \pm 0.7$ & $3.9 \pm 0.7$ & $<0.001$ & $<0.001$ \\
\hline Total cholesterol $(\mathrm{mg} / \mathrm{dL}$, mean $\pm \mathrm{SD})$ & $170.7 \pm 38.2$ & $168.8 \pm 36.8$ & $170.6 \pm 37.4$ & $172.7 \pm 40.1$ & 0.309 & 0.126 \\
\hline LDL (mg/dL, mean $\pm \mathrm{SD})$ & $93.1 \pm 31.8$ & $91.5 \pm 30.9$ & $93.2 \pm 32.4$ & $94.4 \pm 31.9$ & 0.396 & 0.176 \\
\hline${ }^{\mathrm{C}} \mathrm{HDL}(\mathrm{mg} / \mathrm{dL}$, mean $\pm \mathrm{SD})$ & $47.7 \pm 14.6$ & $47.4 \pm 14.3$ & $47.7 \pm 13.6$ & $48.9 \pm 15.9$ & 0.816 & 0.527 \\
\hline${ }^{\mathrm{d}}$ Triglyceride $(\mathrm{mg} / \mathrm{dL}$, mean $\pm \mathrm{SD})$ & $159.2 \pm 95.7$ & $157.0 \pm 89.4$ & $157.7 \pm 85.7$ & $162.8 \pm 110.3$ & 0.607 & 0.361 \\
\hline
\end{tabular}


Table 1 Baseline Characteristics according to Educational attainment (Continued)

\begin{tabular}{|c|c|c|c|c|c|c|}
\hline \multirow[t]{3}{*}{$\mathrm{N}$} & \multirow{3}{*}{$\begin{array}{l}\text { Total } \\
1361\end{array}$} & \multicolumn{3}{|c|}{ Educational attainment } & \multirow[t]{3}{*}{$P$} & \multirow{3}{*}{$\begin{array}{l}P \text { for } \\
\text { trend }\end{array}$} \\
\hline & & \multirow{2}{*}{$\begin{array}{l}\text { College or } \\
\text { beyond } \\
437\end{array}$} & \multirow{2}{*}{$\begin{array}{l}\text { Completed } \\
\text { High school } \\
467\end{array}$} & \multirow{2}{*}{$\begin{array}{l}\text { Lower than } \\
\text { high school } \\
457\end{array}$} & & \\
\hline & & & & & & \\
\hline${ }^{\mathrm{e}} 25-\mathrm{OH}$ vitamin $\mathrm{D}(\mathrm{ng} / \mathrm{mL}$, mean $\pm \mathrm{SD})$ & $18.5 \pm 8.5$ & $19.1 \pm 9.0$ & $18.5 \pm 7.3$ & $17.9 \pm 9.2$ & 0.126 & 0.042 \\
\hline $\begin{array}{l}\text { f } 24 \text { hour urine sodium } \\
(\mathrm{mmol} / \text { day, mean } \pm \mathrm{SD})\end{array}$ & $154.8 \pm 67.7$ & $156.4 \pm 64.7$ & $155.5 \pm 70.2$ & $152.7 \pm 67.9$ & 0.44 & 0.437 \\
\hline \multicolumn{7}{|c|}{ 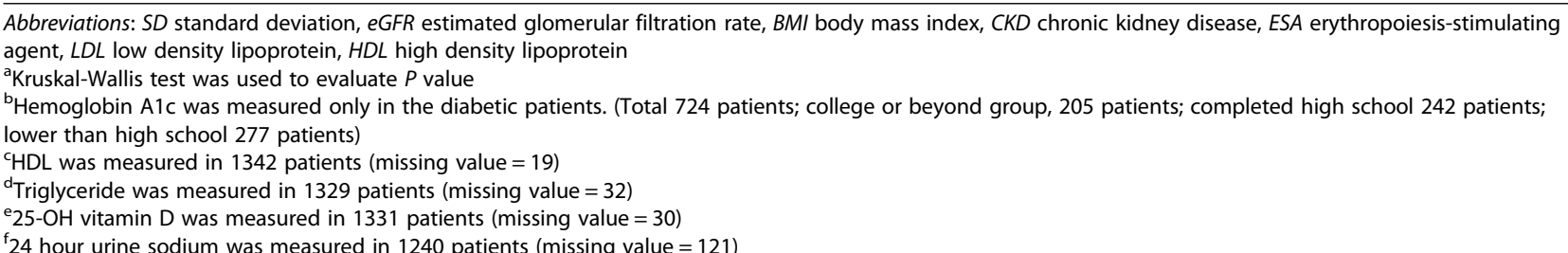 } \\
\hline
\end{tabular}

when anemia included as a covariate in the multi-variable model, the association between the lowest education level and LVH still showed the statistical significance (OR 1.454, 95\% CI 1.042-2.028; $P=0.028$, Table 3). Monthly income level is a risk factor for $\mathrm{LVH}$ in univariate and multivariate analysis adjusted age, sex, body mass index, mean arterial pressure, diabetes, CKD stages and physical activity. However, the inclusion of anemia as a covariates in the multivariable analysis attenuated the relationship between income level and $\mathrm{LVH}$, as shown in the Table 4 (> \$ 4500, reference; \$ 1500 - 4500, OR 1.174, 95\% CI 0.825-1.672, $P=0.373$; < $\$ 1500$, OR $1.415,95 \%$ CI $0.962-2.081, P=0.078)$.

\section{Discussion}

We performed cross-sectional analysis for the relationships between SES and LVH in a CKD population over 50 years of age. LVH was associated with parameters of SES, such as educational attainment and monthly income level, and these associations had graded responses. Additional analyses were conducted to clarify the association between SES and LVH, adjusting for body mass index, sex, age, blood pressure, DM, CKD stages and physical activity. After adjustment, lower education and income level remained a statistically significant determinant of LVH.

Compared with the statistical data of the Organization for Economic Co-operation and Development (OECD) statistics, the proportions of college graduates or above among the subjects at 25-45 years of age, were $69 \%$ for Koreans and $42 \%$ for the average OECD countries, respectively, while among those at 55-64 years of age, they are $18 \%$ in Korea and $26 \%$ in OECD average [20, 21]. Namely, since most of the younger Korean population are college graduates, educational attainment is not a suitable factor for evaluating the SES among younger Korean CKD patients. In such a respect, the present study included only the subjects at the age of 50 or older.
Usually, SES is defined by education, employment, income and poverty, and these indicators could influence one's access to medical care and social support through insurance, housing stability and quality, accessibility to healthy food and degree of stress [10]. Since numerous complex interactions between the selected social determinants of health exist, it is difficult to elucidate the mechanism for the association between SES and many clinical outcomes regarding the incidence, progression of disease and mortality. In particular, low educational attainment has indirect effects on one's understanding of disease and medical treatment. Low income level could affect one's ability to engage in healthy behavior and access to healthcare services. It is important to identify clinical outcomes influenced by SES, because some determinants could be modified by government policies, social support for access medical care, and efforts of physicians and community members.

Explanations for the differences in the burden of LVH between various strata of socio-economic status remain speculative, but there are several potential reasons why lower SES could be an independent risk factor for LVH. First, socioeconomically disadvantaged patients tend to receive less vigorous treatment because of poorer access to medical care [22]. It is well known that early referral to a nephrologist improves clinical outcomes in CKD patients $[23,24]$ due to timely and proper management for prevention of disease progression. The primary system of South Korea health insurance system is the National Health Insurance (NHI), and nearly $96 \%$ of the total Korean population joined this NHI program [25]. It guarantees the basic medical cares including in-patient and out-patient health service, preventive care and prescription drugs, based on the co-payment system [26]. However, with the rapid expansion of enrolled population, the NHI has been under the heavy burdens due to increased medical expenditure [27]. Inevitably, the NHI program had to restrict the range of covered medical services at the possible level [28]. Therefore, some medical services or medical tests at high costs are not 
Table 2 Baseline Characteristics according to Monthly Income Level

\begin{tabular}{|c|c|c|c|c|c|c|}
\hline \multirow[t]{3}{*}{ N } & \multirow{3}{*}{$\begin{array}{l}\text { Total } \\
1361\end{array}$} & \multicolumn{3}{|c|}{ Monthly income } & \multirow[t]{3}{*}{$P$} & \multirow{3}{*}{$\begin{array}{l}P \text { for } \\
\text { trend }\end{array}$} \\
\hline & & $>\$ 4500$ & $\$ 1500$ to $\$ 4500$ & $<\$ 1500$ & & \\
\hline & & 269 & 701 & 391 & & \\
\hline Age (year, mean $\pm S D)$ & $60.9 \pm 6.9$ & $59.0 \pm 6.6$ & $60.6 \pm 6.9$ & $62.8 \pm 6.7$ & $<0.001$ & $<0.001$ \\
\hline Male (N, \%) & $860(63.2)$ & $199(74.0)$ & $433(61.8)$ & $228(58.3)$ & $<0.001$ & $<0.001$ \\
\hline LVH $(\mathrm{N}, \%)$ & $413(30.3)$ & $60(22.3)$ & $205(29.2)$ & $148(37.9)$ & $<0.001$ & $<0.001$ \\
\hline $\mathrm{eGFR}\left(\mathrm{mL} / \mathrm{min} / 1.73 \mathrm{~m}^{2}\right)$ & $45.5 \pm 25.1$ & $49.9 \pm 24.9$ & $46.5 \pm 25.2$ & $40.5 \pm 24.3$ & $<0.001$ & $<0.001$ \\
\hline $\mathrm{BMI}\left(\mathrm{kg} / \mathrm{m}^{2}\right.$, mean $\left.\pm \mathrm{SD}\right)$ & $24.6 \pm 3.1$ & $24.6 \pm 2.8$ & $24.6 \pm 3.2$ & $24.7 \pm 3.2$ & 0.586 & 0.403 \\
\hline CKD stage $(N, \%)$ & & & & & $<0.001$ & $<0.001$ \\
\hline Stage G1 & $112(8.2)$ & $27(10.0)$ & $60(8.6)$ & $25(6.4)$ & & \\
\hline Stage G2 & $226(16.6)$ & $58(21.6)$ & $115(16.4)$ & $53(13.6)$ & & \\
\hline Stage G3a & $250(18.4)$ & $54(20.1)$ & $145(20.7)$ & $51(13.0)$ & & \\
\hline Stage G3b & $322(23.7)$ & $60(22.3)$ & $171(24.4)$ & $91(23.3)$ & & \\
\hline Stage G4 & $352(25.9)$ & $59(21.9)$ & $162(23.1)$ & $131(33.5)$ & & \\
\hline Stage G5 & $99(7.3)$ & $11(4.1)$ & $48(6.8)$ & $40(10.2)$ & & \\
\hline Underlying renal disease $(\mathrm{N}, \%)$ & & & & & $<0.001$ & $<0.001$ \\
\hline Diabetic nephropathy & $429(31.8)$ & $59(22.0)$ & $223(32.2)$ & $147(27.8)$ & & \\
\hline Hypertensive nephropathy & $327(24.2)$ & $71(25.4)$ & $173(25.0)$ & $83(21.3)$ & & \\
\hline Glomerulonephritis & $341(25.3)$ & $88(32.8)$ & $179(25.8)$ & $74(19.0)$ & & \\
\hline Polycystic kidney disease & $140(10.4)$ & $39(14.6)$ & $63(9.1)$ & $38(9.8)$ & & \\
\hline Diabetes mellitus (N, \%) & $571(42.0)$ & $88(32.7)$ & $291(41.5)$ & $192(49.1)$ & & \\
\hline bHemoglobin A1c (\%, mean \pm SD) & $6.9 \pm 1.4$ & $6.8 \pm 1.2$ & $6.9 \pm 1.3$ & $7.0 \pm 1.5$ & 0.293 & 0.125 \\
\hline Hypertension (N, \%) & $1334(98.0)$ & 268 (99.6) & $688(98.1)$ & $378(96.7)$ & 0.069 & 0.041 \\
\hline Systolic blood pressure $(\mathrm{mmHg})$ & $128.84 \pm 16.7$ & $127.0 \pm 14.1$ & $128.3 \pm 17.3$ & $131.0 \pm 17.1$ & $0.005^{\mathrm{a}}$ & 0.001 \\
\hline Diastolic blood pressure (mmHg) & $75.9 \pm 11.2$ & $77.1 \pm 10.2$ & $75.6 \pm 11.3$ & $75.8 \pm 11.5$ & 0.175 & 0.224 \\
\hline Educational attainment (N, \%) & & & & & $<0.001$ & $<0.001$ \\
\hline College graduate or higher & $437(32.1)$ & $163(60.6)$ & $214(30.5)$ & $60(15.3)$ & & \\
\hline Completed high school & $467(34.3)$ & $73(27.1)$ & $279(39.8)$ & $115(29.4)$ & & \\
\hline Lower than high school & 347 (33.6) & $33(12.3)$ & $208(29.7)$ & $216(55.2)$ & & \\
\hline Physical activity (N, \%) & & & & & $<0.001$ & $<0.001$ \\
\hline High & $287(21.1)$ & $55(20.4)$ & $156(22.3)$ & $76(19.4)$ & & \\
\hline Moderate & $536(39.4)$ & $137(50.9)$ & $259(36.9)$ & $140(35.8)$ & & \\
\hline Low & $538(39.5)$ & $77(28.6)$ & $286(40.8)$ & $175(44.8)$ & & \\
\hline Anemia (N, \%) & $662(49.1)$ & $101(37.8)$ & $345(49.8)$ & $216(55.8)$ & $<0.001$ & $<0.001$ \\
\hline ESA $(N, \%)$ & $119(8.7)$ & $12(4.5)$ & $71(10.1)$ & $36(9.2)$ & 0.019 & 0.01 \\
\hline \multicolumn{7}{|l|}{ Serum laboratory values } \\
\hline Hemoglobin (g/dL) & $12.6 \pm 2.0$ & $13.2 \pm 1.9$ & $12.6 \pm 2.0$ & $12.2 \pm 1.9$ & $<0.001$ & $<0.001$ \\
\hline Creatinine (mg/dL) & $1.9 \pm 1.1$ & $1.8 \pm 1.0$ & $1.9 \pm 1.1$ & $2.1 \pm 1.2$ & $<0.001^{\mathrm{a}}$ & $<0.001$ \\
\hline Albumin (g/dL) & $4.2 \pm 0.4$ & $4.2 \pm 0.3$ & $4.1 \pm 0.4$ & $4.1 \pm 0.4$ & $0.003^{\mathrm{a}}$ & 0.001 \\
\hline Calcium (mg/dL) & $9.1 \pm 0.5$ & $9.2 \pm 0.5$ & $9.1 \pm 0.5$ & $9.1 \pm 0.5$ & 0.012 & 0.004 \\
\hline Phosphorus (mg/dL) & $3.7 \pm 0.7$ & $3.6 \pm 0.5$ & $3.7 \pm 0.7$ & $3.8 \pm 0.7$ & $0.003^{a}$ & 0.001 \\
\hline Total cholesterol (mg/dL) & $170.7 \pm 38.2$ & $171.0 \pm 37.0$ & $171.4 \pm 39.4$ & $159.3 \pm 36.8$ & $0.692^{a}$ & 0.508 \\
\hline $\mathrm{LDL}(\mathrm{mg} / \mathrm{dL})$ & $93.1 \pm 31.8$ & $91.1 \pm 32.4$ & $94.4 \pm 31.9$ & $92.1 \pm 31.1$ & 0.281 & 0.851 \\
\hline${ }^{\mathrm{c}} \mathrm{HDL}(\mathrm{mg} / \mathrm{dL})$ & $47.7 \pm 14.6$ & $49.7 \pm 14.5$ & $47.4 \pm 14.2$ & $46.9 \pm 14.3$ & 0.051 & 0.029 \\
\hline${ }^{\mathrm{d}}$ Triglyceride $(\mathrm{mg} / \mathrm{dL})$ & $159.2 \pm 95.7$ & $143.7 \pm 101.3$ & $160.6 \pm 100.0$ & $159.6 \pm 83.4$ & 0.692 & 0.585 \\
\hline
\end{tabular}


Table 2 Baseline Characteristics according to Monthly Income Level (Continued)

\begin{tabular}{|c|c|c|c|c|c|c|}
\hline \multirow[t]{3}{*}{ N } & \multirow[t]{2}{*}{ Total } & \multicolumn{3}{|c|}{ Monthly income } & \multirow[t]{3}{*}{$P$} & \multirow{3}{*}{$\begin{array}{l}P \text { for } \\
\text { trend }\end{array}$} \\
\hline & & $>\$ 4500$ & $\$ 1500$ to $\$ 4500$ & $<\$ 1500$ & & \\
\hline & 1361 & 269 & 701 & 391 & & \\
\hline${ }^{\mathrm{e}} 25-\mathrm{OH}$ vitamin $\mathrm{D}(\mathrm{ng} / \mathrm{mL})$ & $18.5 \pm 8.5$ & $19.4 \pm 8.2$ & $18.7 \pm 9.0$ & $17.6 \pm 7.9$ & 0.019 & 0.006 \\
\hline $\mathrm{f}_{24}$ hour urine sodium ( $\mathrm{mmol} /$ day) & $154.8 \pm 67.7$ & $157.4 \pm 67.5$ & $152.1 \pm 66.5$ & $157.8 \pm 69.6$ & 0.348 & 0.797 \\
\hline
\end{tabular}

Abbreviations: SD standard deviation, eGFR estimated glomerular filtration rate, BMI body mass index, CKD chronic kidney disease, ESA erythropoiesis-stimulating agent, $L D L$ low density lipoprotein, $H D L$ high density lipoprotein

${ }^{a}$ Kruskal-Wallis test was used to evaluate $P$ value

${ }^{\mathrm{b}}$ Hemoglobin A1c was measured only in the diabetic patients. (Total 724 patients; $>\$ 4500,115$ patients; $\$ 1500$ to $\$ 4500,152$ patients; $<\$ 1500,252$ patients)

${ }^{\mathrm{C}} \mathrm{HDL}$ was measured in 1342 patients (missing value $=19$ )

${ }^{\mathrm{d}}$ Triglyceride was measured in 1329 patients (missing value $=32$ )

${ }^{\mathrm{e}} 25-\mathrm{OH}$ vitamin $\mathrm{D}$ was measured in 1331 patients (missing value $=30$ )

$\mathrm{f}_{24}$ hour urine sodium was measured in 1240 patients (missing value $=121$ )

covered by the NHI, rendering them not easily accessible to the individuals at low SES. In addition, low SES leads to decreased understanding of treatment plans and poor compliance, which result in late diagnosis and disease progression.

Second, sympathetic stimulation is one of the mechanisms of LVH in low SES. The role of stress in health has been investigated since the 1950s [29] and the association between stress and cardiovascular disease is well known [30-33]. Sympathetic nervous system activity increases with various environmental factors, including low SES and stress. Lower SES is an important factor in psychosocial stress relative to higher SES [34]. It has

Table 3 The relationship between LVH and educational attainment

\begin{tabular}{|c|c|c|c|c|c|c|}
\hline & \multicolumn{2}{|l|}{ Univariate analysis } & \multicolumn{2}{|l|}{ Multivariate (model 1) } & \multicolumn{2}{|l|}{ Multivariate (model 2) } \\
\hline & $\overline{\mathrm{OR}}(95 \% \mathrm{Cl})$ & $P$ & OR (95\% Cl) & $P$ & OR (95\% Cl) & $P$ \\
\hline \multicolumn{7}{|l|}{ Age } \\
\hline $50-59$ & reference & & reference & & reference & \\
\hline $60-69$ & $1.575(1.218-2.036)$ & 0.001 & 1.409 (1.068-1.859) & 0.015 & $1.442(1.090-1.908)$ & 0.01 \\
\hline$\geq 70$ & $2.468(1.764-3.453)$ & $<0.001$ & $2.246(1.553-3.248)$ & $<0.001$ & $2.308(1.593-3.342)$ & $<0.001$ \\
\hline Sex (vs. men) & $1.797(1.419-2.275)$ & $<0.001$ & $1.863(1.413-2.456)$ & $<0.001$ & $1.885(1.426-2.491)$ & $<0.001$ \\
\hline BMI (reference $\leq 23.0$ ) & $2.254(1.707-2.976)$ & $<0.001$ & $2.401(1.786-3.277)$ & $<0.001$ & $2.457(1.820-3.315)$ & $<0.001$ \\
\hline MAP & $1.018(1.010-1.027)$ & $<0.001$ & $1.018(1.008-1.027)$ & $<0.001$ & $1.019(1.009-1.028)$ & $<0.001$ \\
\hline DM & $1.598(1.266-2.017)$ & $<0.001$ & $1.208(0.934-1.563)$ & 0.151 & $1.159(0.890-1.510)$ & 0.275 \\
\hline \multicolumn{7}{|l|}{ CKD stage } \\
\hline 1 & reference & & reference & & reference & \\
\hline 2 & $1.409(0.794-2.500)$ & 0.241 & $1.464(0.808-2.652)$ & 0.209 & $1.365(0.748-2.490)$ & 0.311 \\
\hline 3a & $1.297(0.735-2.291)$ & 0.369 & $1.293(0.713-2.343)$ & 0.398 & $1.216(0.666-2.223)$ & 0.524 \\
\hline $3 b$ & $1.983(1.157-3.400)$ & 0.013 & $1.776(1.011-3.121)$ & 0.046 & $1.613(0.905-2.875)$ & 0.105 \\
\hline 4 & $3.185(1.878-5.401)$ & $<0.001$ & $3.007(1.723-5.248)$ & $<0.001$ & $2.609(1.443-4.715)$ & 0.002 \\
\hline 5 & 3.680 (1.969-6.877) & $<0.001$ & $3.146(1.630-6.071)$ & 0.001 & $2.645(1.311-5.337)$ & 0.007 \\
\hline \multicolumn{7}{|l|}{ Physical activity } \\
\hline Low & $0.942(0.688-1.290)$ & 0.711 & $0.786(0.560-1.101)$ & 0.161 & $0.817(0.580-1.149)$ & 0.245 \\
\hline Moderate & $1.108(0.812-1.511)$ & 0.518 & $0.747(0.532-1.049)$ & 0.093 & $0.764(0.541-1.078)$ & 0.125 \\
\hline High & reference & & reference & & reference & \\
\hline Anemia & $1.790(1.414-2.266)$ & $<0.001$ & & & $1.219(0.904-1.645)$ & 0.194 \\
\hline \multicolumn{7}{|l|}{ Educational attainment } \\
\hline College graduate or more & reference & & reference & & reference & \\
\hline Completed high school & $1.311(0.971-1.770)$ & 0.077 & $1.150(0.834-1.584)$ & 0.394 & $1.141(0.825-1.579)$ & 0.425 \\
\hline lower than high school & $2.162(1.616-2.892)$ & $<0.001$ & $1.485(1.069-2.063)$ & 0.018 & $1.454(1.042-2.028)$ & 0.028 \\
\hline
\end{tabular}

Abbreviations: $C l$ confidential interval, vs., versus, $B M I$ body mass index, MAP mean arterial pressure, DM diabetes mellitus, CKD Chronic kidney disease;

Model 1: age, sex, BMI, MAP, DM, CKD stage, physical activity, educational attainment, P for trend according to education attainment $=0.017$, $P$ for Hosmer and Lemeshow goodness of fit test $=0.090$

Model 2: age, sex, BMI, MAP, DM, CKD stage, physical activity, anemia, educational attainment, $\mathrm{P}$ for trend according to education attainment $=0.025, P$ for Hosmer and Lemeshow goodness of fit test $=0.086$ 
Table 4 The relationship between LVH and household income level

\begin{tabular}{|c|c|c|c|c|c|c|}
\hline & \multicolumn{2}{|l|}{ Univariate analysis } & \multicolumn{2}{|c|}{ Multivariate (model 3) } & \multicolumn{2}{|l|}{ Multivariate (model 4) } \\
\hline & $\mathrm{OR}(95 \% \mathrm{Cl})$ & $P$ & OR $(95 \% \mathrm{Cl})$ & $P$ & OR $(95 \% \mathrm{Cl})$ & $P$ \\
\hline \multicolumn{7}{|l|}{ Age } \\
\hline $50-59$ & reference & & reference & & reference & \\
\hline $60-69$ & $1.575(1.218-2.036)$ & 0.001 & $1.409(1.067-1.861)$ & 0.016 & $1.444(1.091-1.913)$ & 0.01 \\
\hline$\geq 70$ & $2.468(1.764-3.453)$ & $<0.001$ & $2.255(1.561-3.260)$ & $<0.001$ & $2.318(1.601-3.356)$ & $<0.001$ \\
\hline Sex (vs. men) & $1.797(1.419-2.275)$ & $<0.001$ & $2.027(1.559-2.635)$ & $<0.001$ & $2.044(1.569-2.664)$ & $<0.001$ \\
\hline BMI (reference $\leq 23.0$ ) & $2.254(1.707-2.976)$ & $<0.001$ & $2.438(1.813-3.278)$ & $<0.001$ & $2.496(1.849-3.369)$ & $<0.001$ \\
\hline MAP & $1.018(1.010-1.027)$ & $<0.001$ & $1.017(1.008-1.027)$ & $<0.001$ & $1.018(1.009-1.028)$ & $<0.001$ \\
\hline DM & $1.598(1.266-2.017)$ & $<0.001$ & $1.212(0.837-1.568)$ & 0.143 & $1.159(0.890-1.510)$ & 0.275 \\
\hline \multicolumn{7}{|l|}{ CKD stage } \\
\hline 1 & reference & & reference & & reference & \\
\hline 2 & $1.409(0.794-2.500)$ & 0.241 & $1.443(0.798-2.611)$ & 0.225 & $1.346(0.739-2.451)$ & 0.332 \\
\hline $3 a$ & $1.297(0.735-2.291)$ & 0.369 & $1.254(0.693-2.270)$ & 0.454 & $1.178(0.646-2.148)$ & 0.592 \\
\hline $3 b$ & $1.983(1.157-3.400)$ & 0.013 & $1.770(1.008-3.108)$ & 0.047 & $1.599(0.898-2.847)$ & 0.111 \\
\hline 4 & $3.185(1.878-5.401)$ & $<0.001$ & $2.945(1.689-5.136)$ & $<0.001$ & $2.527(1.399-4.565)$ & 0.002 \\
\hline 5 & $3.680(1.969-6.877)$ & $<0.001$ & $3.034(1.572-5.856)$ & 0.001 & $2.525(1.252-5.091)$ & 0.01 \\
\hline \multicolumn{7}{|l|}{ Physical activity } \\
\hline Low & $0.942(0.688-1.290)$ & 0.711 & $0.791(0.565-1.109)$ & 0.174 & $0.819(0.582-1.152)$ & 0.251 \\
\hline Moderate & $1.108(0.812-1.511)$ & 0.518 & $0.747(0.532-1.409)$ & 0.092 & $0.762(0.540-1.076)$ & 0.122 \\
\hline High & reference & & reference & & reference & \\
\hline Anemia & $1.790(1.414-2.266)$ & $<0.001$ & & & $1.240(0.920-1.672)$ & 0.158 \\
\hline \multicolumn{7}{|l|}{ Income level } \\
\hline$>\$ 4500$ & reference & & reference & & reference & \\
\hline$\$ 1500-4500$ & $1.440(1.035-2.003)$ & 0.03 & $1.230(0.866-1.748)$ & 0.247 & $1.174(0.825-1.672)$ & 0.373 \\
\hline$<\$ 1500$ & $2.122(1.491-3.018)$ & $<0.001$ & $1.471(1.002-2.158)$ & 0.049 & $1.415(0.962-2.081)$ & 0.078 \\
\hline
\end{tabular}

Abbreviations: $\mathrm{Cl}$ confidential interval, vs., versus, $B M I$ body mass index, MAP mean arterial pressure, $D M$ diabetes mellitus, CKD Chronic kidney disease;

Model 3: age, sex, BMI, MAP, DM, CKD stage, physical activity, income level, $P$ for trend according to income level $=0.045, P$ for Hosmer and Lemeshow goodness of fit test $=0.381$

Model 4: age, sex, BMI, MAP, DM, CKD stage, physical activity, anemia, income level, $P$ for trend according to income level $=0.06, P$ for Hosmer and Lemeshow goodness of fit test $=0.111$

been reported that chronic adrenergic stimulation can cause increased left ventricular mass [35]. Moreover, patients with CKD may be unable to adapt easily to stressful situations because stress hormones are metabolized and cleared by the kidney [36]. Patients with CKD may present inappropriate reactions to chronic stress. Thus,
CKD patients with lower SES might be subjected both to more stress, and inappropriate responses to the stress.

Dietary differences between low and high SES groups are also related to LVH. One study reported that increased sodium retention might increase the risk of LVH by activation of the renin-angiotensin system and

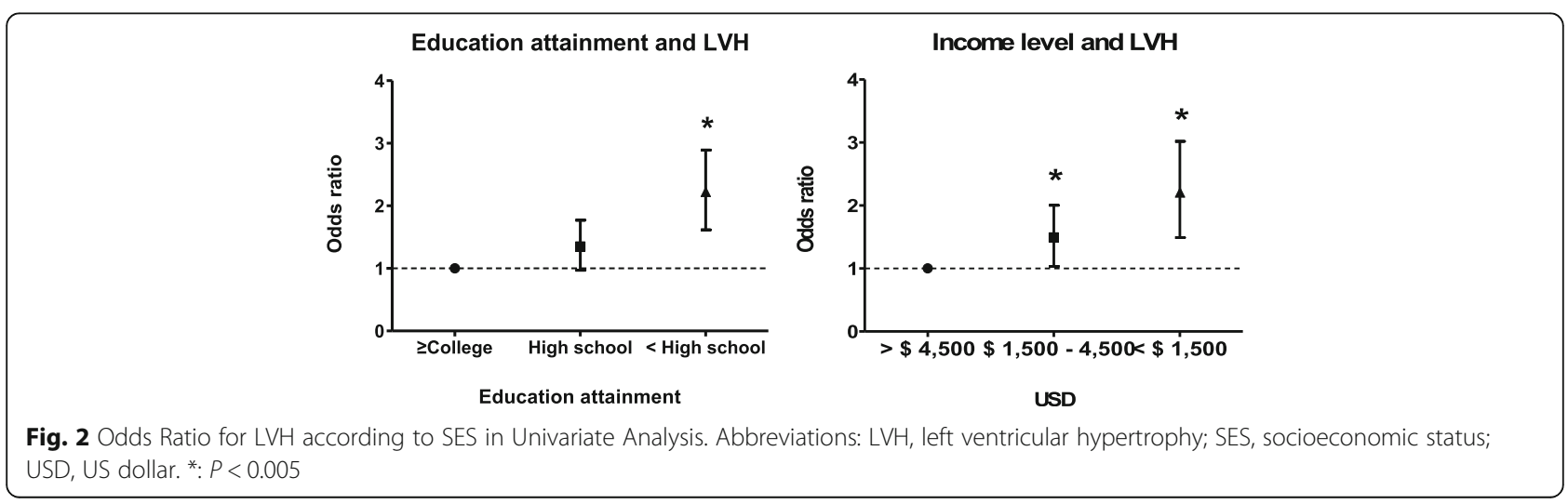




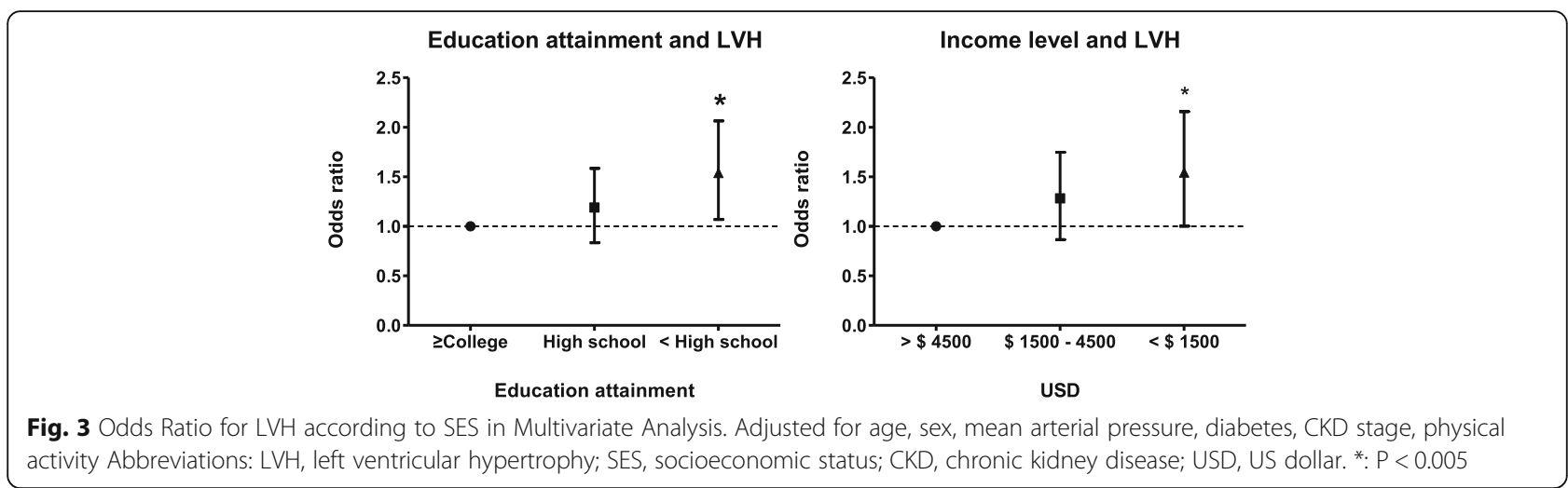

volume expansion [37]. Sodium intake tended to be higher among individuals with low SES [38-40]. In a study carried out among Chinese individuals, more educated participants had a lower intake of salt and soy sauce compared with less educated individuals [41]. This tendency might also be present in CKD populations, and dietary differences could contribute to a greater risk of LVH by sodium intake differences. Although this study did not collect dietary information, we attempted to evaluate sodium intake by measuring 24-h urine $\mathrm{Na}$ excretion, as it might reflect dietary sodium intake [42, 43]. However, our data did not show a significant difference in 24-h urine $\mathrm{Na}$ excretion with respect to educational attainment or income level. Since the main source of sodium intake in the western countries is processed foods $(77 \%$ in the United States and $65-70 \%$ in the United Kingdom), people with low SES are less likely to have access to fresh food, thus, they are more likely to consume more processed food. In other words, the more processed food consumed, the more sodium intake. However, in South Korea, the main sources of salt intake are Kimchi, soup and stew, and these are easily accessible to anyone regardless of SES. Therefore, in Korea, personal salt intake depends more heavily on the personal salt preference, rather than on the SES of the individual. In addition, though adults should consume less than $2000 \mathrm{mg}$ of sodium, or $5 \mathrm{~g}$ of salt per day according to guidelines issued by the World Health Organization (WHO) [44], Korean sodium intake is very high, because Korean food is generally very salty. In fact, looking at KNHANES data, the amount of sodium intake in Korea is $3669 \mathrm{mg}$, far exceeding $2000 \mathrm{mg}$ [45].

Anemia has been shown to be an independent risk factor for LVH in CKD patients [46, 47]. The relationship between SES and anemia has been assessed primarily among adolescents and reproductive-aged women, for whom low SES is an important risk factor for anemia $[48,49]$. Low iron intake among low SES populations also has been well established [50, 51]. In our subjects, anemia was more prevalent in the lowest educational group. Based on the above findings, we hypothesized that low SES might be associated with anemia, which, in turn, might lead to LVH. Our study showed that inclusion of anemia as a covariate in the multivariate analysis attenuated the significant association between low income and LVH. This means that anemia could partly be a contributing factor to the development of LVH in the CKD subjects in lower SES. However, even after adjustment for anemia as a covariate, low educational attainment still remained an independent risk factor for $\mathrm{LVH}$ (lower than high school, OR 1.454, 95\% CI 1.042-2.028, $P=0.028$; Table 3 ). Namely, other social determinants affected by SES but not included in the analysis might contribute to LVH. Further studies are warranted for elucidating the interconnected mechanism underlying the association between SES and LVH.

Our study tried to evaluate the economic status more accurately by surveying the individual or household monthly income, rather than an area income. However, relative to the association between education level and LVH, the association between monthly income level and LVH was somewhat attenuated after adjustment for anemia. It could be speculated that regular monthly income does not represent the overall economic status of a subject, particularly for an elderly or retired person with ample savings or real estate, but no regular monthly income. Therefore, a better parameter that could assess the economic status of an individual subject needs to be investigated.

This study is the first, to our knowledge, to elucidate that lower SES is an independent risk factor of LVH among CKD population. However, several limitations exist. First, although LVH is influenced by many factors, and we tried to adjust as many factors as possible related with health and dietary behavior, there still remains a possibility for a residual confounder. Since this study was conducted as a cross-sectional analysis, we could not determine causality between SES and LVH. However, longitudinal follow-up of the same study subjects will show us the causal relationship between SES and CV outcome. Second, we have excluded those who did not 
respond to the self-questionnaire and LV mass measurement. Because these patients are more likely to have poor compliance, it might influence the results. In addition, because information on the income and educational status was based only on the self-report, there may be a reporting bias. Although echocardiography was performed at each of nine participating centers, the data coordinating center of the KNOW-CKD Study collected each measurement parameter, calculated LV mass index, and relative wall thickness and classified LV geometry following a uniform criteria from the American Society of Echocardiography [17]. Lastly, the study enrolled only ethnic Korean patients; thus, it cannot provide information on the ethnic disparities in CKD.

\section{Conclusions}

In summary, we identified a novel relationship between SES and LVH in CKD patients. Lower SES, defined by educational attainment and monthly income level, is an independent factor for LVH among CKD patients. Further studies are needed to explore the causal relationships between the SES and adverse cardiovascular outcomes in the CKD population, to address factors related to socio-environmental causes of LVH and to develop preventive strategies for $\mathrm{CV}$ mortality in patients with kidney disease. Such efforts will minimize socio-economic disparities, and improve CV outcomes for patients with CKD.

\section{Abbreviations \\ Cl: Confidential interval; CKD: Chronic kidney disease; CKD-EPI: Chronic Kindey Disease Epidemiology Collaboration; CV: Cardiovascular; DM: Diabetes mellitus; eGFR: Estimated glomerular filtration rate; ESRD: End-stage renal disease; HTN: Hypertension; KNHANES: Korea National Health and Nutrition Examination Survey; KNOW-CKD: KoreaN Cohort Study for Outcome in Patients With Chronic Kidney Disease; LV: Left ventricle; LVH: Left ventricular hypertrophy; LVIDd: Left ventricular internal diameter end diastole; \\ MET: Metabolic equivalent of task; NHI: National Health Insurance; OR: Odds ratio; PWTd: Posterior wall thickness in diastole; SES: Socioeconomic status; SWTd: Septum wall thickness in diastole; WHO: World Health Organization}

\section{Acknowledgements}

We are deeply grateful to the Seung-Mi Lee and A Ram Lee who made a great commitment to building this cohort.

\section{Funding}

This study was supported by the research program funded by the Korea Center for Disease Control and Prevention (2011E3300300, 2012E3301100, 2013E3301600, 2013E3301601, 2013E3301602, and 2016E3300200). The study is supervised by the CKD Advisory Committee composed of members from the KCDC and the Korean Society of Nephrology (KSN, NCT01630486 at http://www.clinicaltrials.gov). The funders had no role in study design, data collection or analysis, decision to publish, or preparation of the manuscript.

\section{Availability of data and materials}

The dataset generated and analysed during the current study are available in the http://www.know-ckd.org/ckd/main/main.html.

\section{Authors' contributions}

All authors were involved in drafting this article and/or revising it critically for important intellectual content, and all authors approved the final version to be submitted for publication. Study design: CA, KO, EK; acquisition of data: HJK, MH, SWK, KL, SS, TY, WC; data analysis: JL, MH, EK, KO; writing the manuscript: EK, KO; review, revision, and final approval: all authors.

\section{Ethics approval and consent to participate}

Ethics approval obtained from the Institutional Review Board at each participating clinical center - i.e., Seoul National University Hospital (1104089-359), Seoul National University Bundang Hospital (B-1106/129-008), Yonsei University Severance Hospital (4-2011-0163), Kangbuk Samsung Medical Center (2011-01-076), Seoul St. Mary's Hospital (KC11OIMI0441), Gil Hospital (GIRBA2553), Eulji General Hospital (201105-01), Chonnam National University Hospital (CNUH-2011-092), and Pusan Paik Hospital (11-091) in 2011. Written informed consent was obtained from the participants.

\section{Consent for publication}

Not applicable.

\section{Competing interests}

The authors declare that they have no competing interests.

\section{Publisher's Note}

Springer Nature remains neutral with regard to jurisdictional claims in published maps and institutional affiliations.

\section{Author details}

${ }^{1}$ Department of Internal Medicine, Seoul National University College of Medicine, Seoul, Korea. ${ }^{2}$ School of Medicine, Inha University, Incheon, Korea. ${ }^{3}$ Department of Prevention and Management, Inha University Hospital, Incheon, Korea. ${ }^{4}$ Department of Internal Medicine, Dongguk University Gyeongju Hospital, Gyeongju, Korea. ${ }^{5}$ Department of Internal Medicine, Pusan National University Hospital, Pusan, Korea. ${ }^{6}$ Department of Internal Medicine, Chonnam National University Medical School, Gwangju, Korea. ${ }^{7}$ Division of Nephrology, Kangbuk Samsung Hospital, Sungkyunkwan University School of Medicine, Seoul, Korea. ${ }^{8}$ Department of Internal Medicine, Nowon Eulji Medical Center, Eulji University, Seoul, Korea. ${ }^{9}$ Department of Internal Medicine, Yonsei University College of Medicine, Seoul, Korea. ${ }^{10}$ Department of Internal Medicine, Gachon University Gil Medical Center, Gachon University School of Medicine, Incheon, Korea.

Received: 4 October 2017 Accepted: 7 August 2018

Published online: 16 August 2018

References

1. Adler NE, Boyce WT, Chesney MA, Folkman S, Syme SL. Socioeconomic inequalities in health. No easy solution. Jama. 1993;269(24):3140-5.

2. Adler NE, Ostrove JM. Socioeconomic status and health: what we know and what we don't. Ann N Y Acad Sci. 1999;896:3-15.

3. Marmot MG, Rose G, Shipley M, Hamilton PJ. Employment grade and coronary heart disease in British civil servants. J Epidemiol Community Health. 1978:32(4):244-9.

4. Adelstein AM, Bulusu L. Changing social-class distribution of heart disease. Br Med J. 1978:2(6154):1785.

5. Choi Al, Weekley CC, Chen SC, Li S, Tamura MK, Norris KC, Shlipak MG. Association of educational attainment with chronic disease and mortality: the kidney early evaluation program (KEEP). Am J Kidney Dis. 2011;58(2):228-34.

6. Meadows P. Variation of diabetes mellitus prevalence in general practice and its relation to deprivation. Diabet Med. 1995;12(8):696-700.

7. Berg JW, Ross R, Latourette HB. Economic status and survival of cancer patients. Cancer. 1977:39(2):467-77.

8. Bradley CJ, Given CW, Roberts C. Race, socioeconomic status, and breast cancer treatment and survival. J Natl Cancer Inst. 2002;94(7):490-6.

9. Bello AK, Peters J, Rigby J, Rahman AA, El Nahas M. Socioeconomic status and chronic kidney disease at presentation to a renal service in the United Kingdom. Clin J Am Soc Nephrol. 2008;3(5):1316-23.

10. Norton JM, Moxey-Mims MM, Eggers PW, Narva AS, Star RA, Kimmel PL, Rodgers GP. Social determinants of racial disparities in CKD. J Am Soc Nephrol. 2016;27(9):2576-95

11. Korean Society of Nephrology. Current Renal Replacement Therapy in Korea - Insan Memorial Dialysis Registry. http://www.ksn.or.kr/file/sinchart/ 982988834 eddaa4e5 2016 EC9B90EAB3A0.pdf. Accessed 20 Sep 2017.

12. Middleton RJ, Parfrey PS, Foley RN. Left ventricular hypertrophy in the renal patient. J Am Soc Nephrol. 2001;12(5):1079-84.

13. Parfrey PS, Foley RN, Harnett JD, Kent GM, Murray DC, Barre PE. Outcome and risk factors for left ventricular disorders in chronic uraemia. Nephrol Dial Transplant. 1996;11(7):1277-85. 
14. Rodriguez CJ, Sciacca RR, Diez-Roux AV, Boden-Albala B, Sacco RL, Homma S, DiTullio MR. Relation between socioeconomic status, race-ethnicity, and left ventricular mass: the northern Manhattan study. Hypertension. 2004;43(4):775-9.

15. Oh KH, Park SK, Park HC, Chin HJ, Chae DW, Choi KH, Han SH, Yoo TH, Lee K, Kim YS, et al. KNOW-CKD (KoreaN cohort study for outcome in patients with chronic kidney disease): design and methods. BMC Nephrol. 2014;15:80.

16. Levey AS, Stevens LA, Schmid CH, Zhang YL, Castro AF 3rd, Feldman HI, Kusek JW, Eggers P, Van Lente F, Greene T, et al. A new equation to estimate glomerular filtration rate. Ann Intern Med. 2009;150(9):604-12.

17. Lang RM, Bierig M, Devereux RB, Flachskampf FA, Foster E, Pellikka PA, Picard MH, Roman MJ, Seward J, Shanewise JS, et al. Recommendations for chamber quantification: a report from the American Society of Echocardiography's Guidelines and Standards Committee and the Chamber Quantification Writing Group, developed in conjunction with the European Association of Echocardiography, a branch of the European Society of Cardiology. J Am Soc Echocardiogr. 2005;18(12):1440-63.

18. de Simone G, Devereux RB, Daniels SR, Koren MJ, Meyer RA, Laragh JH. Effect of growth on variability of left ventricular mass: assessment of allometric signals in adults and children and their capacity to predict cardiovascular risk. J Am Coll Cardiol. 1995;25(5):1056-62.

19. Glassock RJ, Pecoits-Filho R, Barberato SH. Left ventricular mass in chronic kidney disease and ESRD. Clin J Am Soc Nephrol. 2009;4(Suppl 1):S79-91.

20. Statistics Korea. National education level (Population distribution by educational attainment). http://kostat.go.kr/portal/eng/index.action. Accessed 20 Sep 2017

21. OECD. Education at a Glance 2017: OECD indicators. https://www.hm.ee/ sites/default/files/eag2017_eng.pdf. Accessed 20 Sep 2017.

22. Vart P, Gansevoort RT, Crews DC, Reijneveld SA, Bultmann U. Mediators of the association between low socioeconomic status and chronic kidney disease in the United States. Am J Epidemiol. 2015;181(6):385-96.

23. Kim DH, Kim M, Kim H, Kim YL, Kang SW, Yang CW, Kim NH, Kim YS, Lee JP. Early referral to a nephrologist improved patient survival: prospective cohort study for end-stage renal disease in Korea. PLoS One. 2013;8(1):e55323.

24. Wavamunno MD, Harris DC. The need for early nephrology referral. Kidney Int Suppl. 2005;94:S128-32.

25. Moon S, Shin J. Performance of universal health insurance: lessons from South Korea. World Health Popul. 2007;9(2):95-113.

26. Liaropoulos LL. Health services financing in Greece: a role for private health insurance. Health Policy. 1995;34(1):53-62.

27. Kwon S, Reich MR. The changing process and politics of health policy in Korea. J Health Polit Policy Law. 2005;30(6):1003-26.

28. Shin J. Private health insurance in South Korea: an international comparison. Health Policy. 2012;108(1):76-85.

29. Selye H. The Stess of Life. New York: McGraw-Hill; 1956.

30. Theorell T, Karasek RA. Current issues relating to psychosocial job strain and cardiovascular disease research. J Occup Health Psychol. 1996;1(1):9-26.

31. Dimsdale JE. Psychological stress and cardiovascular disease. J Am Col Cardiol. 2008;51(13):1237-46.

32. Everson-Rose SA, Lewis TT. Psychosocial factors and cardiovascular diseases. Annu Rev Public Health. 2005;26:469-500.

33. Sternberg EM, Chrousos GP, Wilder RL, Gold PW. The stress response and the regulation of inflammatory disease. Ann Intern Med. 1992;117(10):854-66.

34. Baum A, Garofalo JP, Yali AM. Socioeconomic status and chronic stress. Does stress account for SES effects on health? Ann N Y Acad Sci. 1999;896:131-44.

35. Rapaport E. Pathophysiological basis of ventricular hypertrophy. Eur Heart J. 1982;3 Suppl A:29-33.

36. Cukor D, Cohen SD, Peterson RA, Kimmel PL. Psychosocial aspects of chronic disease: ESRD as a paradigmatic illness. J Am Soc Nephrol. 2007; 18(12):3042-55.

37. de Simone G, Devereux RB, Roman MJ, Schlussel Y, Alderman MH, Laragh $\mathrm{JH}$. Echocardiographic left ventricular mass and electrolyte intake predict arterial hypertension. Ann Intern Med. 1991;114(3):202-9.

38. Vlismas K, Stavrinos V, Panagiotakos DB. Socio-economic status, dietary habits and health-related outcomes in various parts of the world: a review. Cent Eur J Public Health. 2009;17(2):55-63.

39. Darmon N, Drewnowski A. Does social class predict diet quality? Am J Clin Nutr. 2008;87(5):1107-17

40. Grimes CA, Campbell KJ, Riddell LJ, Nowson CA. Is socioeconomic status associated with dietary sodium intake in Australian children? A crosssectional study. BMJ Open. 2013;3(2). https://bmjopen.bmj.com/content/3/ 2/e002106.long.
41. Tian $H G, H u$ G, Dong QN, Yang XL, Nan Y, Pietinen P, Nissinen A. Dietary sodium and potassium, socioeconomic status and blood pressure in a Chinese population. Appetite. 1996;26(3):235-46.

42. Ortega RM, Lopez-Sobaler AM, Ballesteros JM, Perez-Farinos N, RodriguezRodriguez E, Aparicio A, Perea JM, Andres P. Estimation of salt intake by 24 h urinary sodium excretion in a representative sample of Spanish adults. $\mathrm{Br}$ J Nutr. 2011;105(5):787-94.

43. Ribic CH, Zakotnik JM, Vertnik L, Vegnuti M, Cappuccio FP. Salt intake of the Slovene population assessed by $24 \mathrm{~h}$ urinary sodium excretion. Public Health Nutr. 2010;13(11):1803-9.

44. Organization WH. Diet, nutrition and the prevention of chronic diseases (WHO technical report series, no. 916). Geneva: World Health Organization Press; 2003.

45. How to quantify salt intake in certain patients [https://www.escardio.org/ Journals/E-Journal-of-Cardiology-Practice/Nolume-10/How-to-quantify-saltintake-in-certain-patients]. Accessed 20 Sept 2017.

46. Parfrey PS, Foley RN. The clinical epidemiology of cardiac disease in chronic renal failure. J Am Soc Nephrol. 1999;10(7):1606-15.

47. Astor BC, Arnett DK, Brown A, Coresh J. Association of kidney function and hemoglobin with left ventricular morphology among African Americans: the atherosclerosis risk in communities (ARIC) study. Am J Kidney Dis. 2004; 43(5):836-45.

48. Kim JY, Shin S, Han K, Lee KC, Kim JH, Choi YS, Kim DH, Nam GE, Yeo HD, Lee $H G$, et al. Relationship between socioeconomic status and anemia prevalence in adolescent girls based on the fourth and fifth Korea National Health and nutrition examination surveys. Eur J Clin Nutr. 2014;68(2):253-8.

49. Nguyen PH, Gonzalez-Casanova I, Nguyen H, Pham H, Truong TV, Nguyen S, Martorell R, Ramakrishnan U. Multicausal etiology of anemia among women of reproductive age in Vietnam. Eur J Clin Nutr. 2015;69(1):107-13.

50. Shimakawa T, Sorlie P, Carpenter MA, Dennis B, Tell GS, Watson R, Williams $\mathrm{OD}$. Dietary intake patterns and sociodemographic factors in the atherosclerosis risk in communities study. ARIC study investigators. Prev Med. 1994;23(6):769-80.

51. Hulshof KF, Brussaard JH, Kruizinga AG, Telman J, Lowik MR. Socio-economic status, dietary intake and $10 \mathrm{y}$ trends: the Dutch National Food Consumption Survey. Eur J Clin Nutr. 2003;57(1):128-37.

\section{Ready to submit your research? Choose BMC and benefit from:}

- fast, convenient online submission

- thorough peer review by experienced researchers in your field

- rapid publication on acceptance

- support for research data, including large and complex data types

- gold Open Access which fosters wider collaboration and increased citations

- maximum visibility for your research: over $100 \mathrm{M}$ website views per year

At BMC, research is always in progress.

Learn more biomedcentral.com/submissions 